\title{
新型抗糖尿病铬(III)配合物的合成和生物活性及机理探究
}

\author{
董金龙 ${ }^{*, a, b}$ 沈腊珍 ${ }^{a}$ 文斌 $^{a}$ 宋珍 ${ }^{a}$ 冯俊杰 ${ }^{a}$ \\ 梁钢 ${ }^{c} \quad$ 刘斌 $b$ 杨斌盛 $*, b$ \\ $\left({ }^{a}\right.$ 太原师范学院化学系 晋中 030619) \\ $\left({ }^{b}\right.$ 山西大学分子科学研究所 化学生物学与分子工程教育部重点实验室 太原 030006) \\ ( ${ }^{c}$ 山西医科大学第一医院病理科 太原 030001)
}

\begin{abstract}
摘要 为了寻找新型的抗糖尿病分子, 以苯乙双胍为前体, 制备了苯乙双胍铬(III)配合物. 通过元素分析、摩尔电导率、 电喷雾质谱、红外光谱、紫外可见光谱和核磁共振波谱对配合物的结构进行了表征, 并研究了配合物在不同温度、不 同 $\mathrm{pH}$ 值下溶液的稳定性、与 $\mathrm{H}_{2} \mathrm{O}_{2}$ 的反应性和形貌. 同时, 构建了糖尿病 C57 小鼠模型, 研究了其生物活性和毒性. 结 果证明，配合物对其活性指标均有好的抑制作用，保留了苯乙双胍的降糖性质，对机体是无毒的，并且通过 MTT (3-(4,5-二甲基噻唑-2)-2,5-二苯基四氮唑澳盐)实验说明配合物的生物相容性较好, 实现了金属配合物降糖与控脂的多 功能化应用. 在此基础上, 通过光谱法研究了配合物与胰高血糖素的相互作用, 表明配合物对胰高血糖素是静态猝灭 以较强的作用力结合, 条件结合常数为 $1.29 \times 10^{5} \mathrm{~L}^{-} \mathrm{mol}^{-1}$, 结合位点数约为 1 , 初步提出了配合物的新的降糖机制. 关键词＼cjkstart苯乙双胍铬；糖尿病；生物活性；胰高血糖素；毒性
\end{abstract}

\section{Synthesis of A Novel Anti-diabetes Chromium(III) Complex and Investigation of Its Biological Activity and Mechanism}

\author{
Dong, Jinlong*,a, Shen, Lazhen ${ }^{a} \quad$ Wen, $B^{*}{ }^{a} \quad$ Song, Zhen ${ }^{a} \quad$ Feng, Junjie ${ }^{a}$ \\ Liang, Gang ${ }^{c} \quad$ Liu, Bin ${ }^{b} \quad$ Yang, Binsheng*,b \\ $\left({ }^{a}\right.$ Department of Chemistry, Taiyuan Normal University, Jinzhong 030619, China) \\ $\left({ }^{b}\right.$ Institute of Molecular Science, Key Laboratory of Chemical Biology of Molecular \\ Engineering of Education Ministry, Shanxi University, Taiyuan 030006, China) \\ ( ${ }^{c}$ Department of Pathology, First Hospital of Shanxi Medical University, Taiyuan 030001, China)
}

\begin{abstract}
In order to search for novel anti-diabetes molecules, phenformin (Phf) was used as precursors to prepare chromium(III) complex $\left[\mathrm{Cr}(\mathrm{Phf})_{3}\right] \mathrm{Cl}_{3}$ at room temperature. The complex was characterized by elemental analysis (EA), molar conductivity (MC), electrospray ionization mass spectrometry (ESI-MS), infrared (IR), UV-vis and NMR spectroscopy, respectively. In this work, the stability of complex solutions at different temperatures and $\mathrm{pH}$ values, reactivity with $\mathrm{H}_{2} \mathrm{O}_{2}$ were discussed in detail. The morphology and thermal studies of the complex were also investigated. Meanwhile, C57 diabetic mouse model induced by diet combined with streptozocin (STZ) was established to explore its biological activity from the aspects of fasting blood glucose (FBG), fasting serum insulin (FINS), total cholesterol (TC), triglyceride (TG), high density lipoprotein cholesterol (HDL-c), low density lipoprotein cholesterol (LDL-c) levels, and oral toxicity. Afterwards, in order to explore the biological hypoglycemic mechanism of the complex, the interaction between the complex and glucagon was studied at $(37 \pm 0.5){ }^{\circ} \mathrm{C}$ in Phosphate Buffer Saline (PBS) buffer at $\mathrm{pH} 7.4$ by fluorescence spectra, which the conditional binding constant $K$ is $1.29 \times 10^{5} \mathrm{~L}^{\circ} \mathrm{mol}^{-1}$, and the number of binding sites $n$ is about 1 . As a result, the interaction between the complex and glucagon was static quenching. The complex which retained the glucose-lowering properties of Phf exhibited good physical and chemical properties, beneficial function on blood glucose and lipid metabolism for Type II Diabetes mellitus (T2DM). The glucose-lowering mechanism of the complex was proposed, and the multi-functional application of metal complex in glucose-lowering and lipid-controlling was also achieved. Furthermore, oral toxicity results showed that the complex had no toxicity on all organs of mice. Methyl Thiazolyl Tetrazolium (MTT) assays also showed that the complex exhibited lower cytotoxicity than the positive control $\mathrm{CrCl}_{3}$ and $\mathrm{Phf}$. Taken together, these results demonstrated that the non-toxic $\left[\mathrm{Cr}(\mathrm{Phf})_{3}\right] \mathrm{Cl}_{3}$ complex might be a potential candidate for novel anti-diabetic drug development. It may also provide a new
\end{abstract}

\footnotetext{
*E-mail: dongjinlong20123@163.com; yangbs@sxu.edu.cn

Received July 2, 2020; published September 3, 2020.

Supporting information for this article is available free of charge via the Internet at http://sioc-journal.cn.
}

Project supported by the the National Natural Science Foundation of China (Nos. 21571117, 21701121), the Natural Science Foundation of Shanxi Province (No. 201801D121064), the Science and Technology Innovation Project of Colleges and Universities in Shanxi Province (No. 2019L0818), and the Innovation Project for College Students in Shanxi Province (No. CXCY1905).

项目受国家自然科学基金(Nos. 21571117, 21701121)、山西省自然科学基金(No. 201801D121064)、山西高校科技项目(No. 2019L0818)和太原师范学 院大学生创新创业项目(No. CXCY1905)资助. 


\section{1 引言}

糖尿病是一种导致严重并发症的慢性疾病, 影响全 球超过 4.25 亿人 ${ }^{[1]}$. 随着人们生活质量的提高、饮食结 构的改变和体力劳动的减少, 二型糖尿病 (T2DM)成为 继癌症、心血管疾病之后的第三大慢性非传染性疾病, 其发病率在全球呈逐年递增的趋势 ${ }^{[2-4]}$. T2DM 极大地影 响着人们的健康, 已成为当今威胁人类健康的重要疾 病, 因此, 有效地对之予以防治具有十分重要的意义.

早在 20 世纪 50 年代, Mertz 和 Schwartz 提出铬(III) 是葡萄糖耐量因子(glucose tolerance factor, GTF)的中心 活性组分, GTF 可增强组织对胰岛素的敏感性, 促进体 内葡萄糖转化为其它物质 ${ }^{[5,6]}$. 值得注意的是, 随着年龄 的增长三价铬离子会慢慢随体液流失, 进而导致 GTF 分子结构的不完整, GTF 的生物学效应也会减弱甚至丧 失 ${ }^{[7]}$, 因此, 外源性的补充铬(III)对机体糖代谢具有较 重要的意义. 目前, 市场上销售的铬酵母片、吡啶甲酸 铭等各种营养铬(III)产品, 种类繁多, 非常畅销. 无机 铬(III)吸收较差, 而三价铬可以与多种氨基酸、有机物、 药物等形成配合物, 例如: 吡啶甲酸铬、烟酸铬、苯丙 氨酸铬、柠檬酸铬、水杨酸铬等, 提高吸收率的同时, 可 以发挥多种生物学效应, 并且有利于改善糖耐量、调节 血脂和预防心脑血管等疾病 ${ }^{[8-10]}$. 因此, 开发新型功能 性营养补铬(III)产品具有战略性的意义.

本课题组在水杨酸铬(III)研发的基础上 ${ }^{[11-14]}$, 选用 双胍类降血糖药苯乙双胍为配体，与 $\mathrm{Cr}(\mathrm{III})$ 配位制备了 新型苯乙双胍铬(III)配合物, 对其结构进行了表征并研 究了其稳定性和抗氧化性; 进一步通过链艮佐菌素诱导 C57 小鼠致糖尿病鼠模型, 进行了 12 周的药物干预实验 研究了其生物活性, 取得了较好的降糖效果. 在此研究 基础上, 为更进一步探究该化合物的降糖机制, 采用与 糖尿病病因相关的胰高血糖素 (glucagon) 为靶点 ${ }^{[15]}$, 研 究了配合物与胰高血糖素的相互作用, 旨在为其在糖尿 病中的辅助治疗提供新的方案, 未来也有可能实现铬 (III)配合物临床药物开发和应用的突破, 本工作研究思 路如图 1 所示.

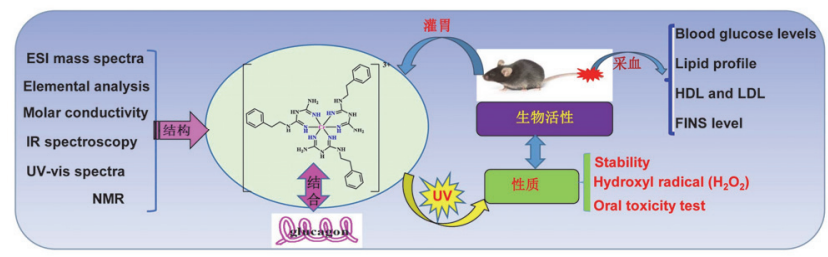

图 1 研究思路框图

Figure 1 Conceptual framework

\section{2 结果与讨论}

\section{1 配合物的表征与结构}

配合物的合成是通过盐酸苯乙双胍在强碱环境中, 与三氯化铬按物质的量比 $3: 1$ 在室温下进行反应, 生 成了红色固体, 经过乙醚乙醇洗涤生成较纯的红色配合 物. C、H、N 含量通过 EURO EA3000 元素分析仪测定, 由分子式 $\mathrm{CrC}_{30} \mathrm{H}_{45} \mathrm{~N}_{15} \mathrm{Cl}_{3}$ 计算结果(\%): C 46.55, H 5.86, N 27.14; 实验结果(\%): C 46.08, H 5.91, N 27.03, 理论 计算值与实验值基本一致; 摩尔电导率测试值为 131.7 $\mu \mathrm{s} \bullet \mathrm{cm}^{-1}\left(10^{-3} \mathrm{~mol} \cdot \mathrm{L}^{-1}, \mathrm{DMSO}\right)$, 表明配合物是多电荷的 物质; 电喷雾质谱图上(见图 S1) ESI-MS $(\mathrm{m} / \mathrm{z})$ : 理论计 算结果: $222.4462[\mathrm{M} / 3]^{+}$, 实验检测结果: 222.1349 $[\mathrm{M} / 3]^{+}$, 二者结果基本吻合.

红外图谱(IR, 见图 S2), 在苯乙双胍图上高频区 $3318 \sim 3164 \mathrm{~cm}^{-1}$ 处强的单、双峰分别是 $\mathrm{NH}_{2}, \mathrm{NH}$ 伸缩 振动所致, 与 $\mathrm{Cr}(\mathrm{IIII})$ 发生配位形成配合物后, 在 3333 $\mathrm{cm}^{-1}$ 处呈现出一个宽且尖的峰, 这是由于 $\mathrm{Cr}(\mathrm{III})$ 与 $\mathrm{NH}$ 配位发生了缔合作用，而且在这个区域未出现 $\mathrm{H}_{2} \mathrm{O}$ 的特 征峰; 配合物图上在 1511,1583 和 $1664 \mathrm{~cm}^{-1}$ 处的吸收 峰, 推断 $\mathrm{Cr}(\mathrm{III})$ 与 $\mathrm{NH}$ 上的 $\mathrm{N}$ 进行了配位; 最后, 配合 物图上指纹区 $420 \mathrm{~cm}^{-1}$ 处出现两个新的伸缩振动是 $\mathrm{Cr}-\mathrm{N}$ 的配位键 ${ }^{[16]}$.

配合物的 ${ }^{1} \mathrm{H}$ NMR 和 ${ }^{13} \mathrm{C}$ NMR 图谱见支撑信息图 $\mathrm{S} 3$ 和 S4. 因为三价铬 $\mathrm{Cr}$ 的顺磁性, ${ }^{1} \mathrm{H}$ NMR 图上在 $\delta$ 7.22 7.59 呈现出宽峰, 是配体苯环上的 $\mathrm{H} ; \delta 5.44$ 和 2.78 处的强峰分别是胺基和甲基上的 $\mathrm{H} ; \delta 2.51$ 和 3.35 的强峰是氞代 DMSO 的峰. 在 ${ }^{13} \mathrm{C}$ NMR 图上, $\delta$ 55.47, $79.75,126.69$ 和 128.90 处的三个弱峰依次归属配体上的 甲基、苯环和胺基上的 C.

图 2(a)是配合物的紫外-吸收图谱, 苯乙双胍在 210 和 $236 \mathrm{~nm}$ 处有两个紫外吸收, 而配合物在 $220 \mathrm{~nm}$ 右侧 有一个约 $232 \mathrm{~nm}$ 的肩峰, 这是 $\mathrm{Cr}(\mathrm{III})$ 与苯乙双胍配位 后发生轻微的蓝移, 谱带的移动证明配体上的电子云向 $\mathrm{Cr}$ (III)的空 $\mathrm{d}$ 轨道发生移动; 此外, 381 和 $500 \mathrm{~nm}$ 处的两 个宽吸收峰是配合物 $\mathrm{Cr}(\mathrm{III})$ 的 d-d 跃迁峰, 与 $\mathrm{Cr}\left(\mathrm{H}_{2} \mathrm{O}\right)_{6}{ }^{3+}$ 跃迁峰 406 和 $574 \mathrm{~nm}$ 对比 ${ }^{[14]}$, 分别发生了 25 和 $74 \mathrm{~nm}$ 的蓝移, 可推断 $\mathrm{Cr}(\mathrm{III})$ 与双胍上的 $\mathrm{NH}$ 发生了双齿配位. 同时, 381 和 $500 \mathrm{~nm}$ 的两个跃迁峰被分别归属于 ${ }^{4} \mathrm{~A}_{2 \mathrm{~g}} \rightarrow{ }^{4} \mathrm{~T}_{2 \mathrm{~g}}(\mathrm{~F})$ 和 ${ }^{4} \mathrm{~A}_{2 \mathrm{~g}} \rightarrow{ }^{4} \mathrm{~T}_{1 \mathrm{~g}}(\mathrm{P})$, 这也证明了三价铬的假 八面体 $\left[\mathrm{CrN}_{6}\right]$ 构型 ${ }^{[17,18]}$.

综上, 经过元素分析、电导率、电喷雾质谱、红外、 核磁和紫外的细致分析, 拟推断苯乙双胍铬(III)的结构 式：一个铬 (III) 与三个苯乙双胍配位形成稳定的 $\mathrm{Cr}[\mathrm{Phf}]^{3+}$ 的多电荷离子, 三个 $\mathrm{Cl}^{-}$作为平衡离子, 如图 2(b)所示, 而此结构与杨小平等 ${ }^{[10]}$ 研究的苯丙氨酸铬的 
结构相近.
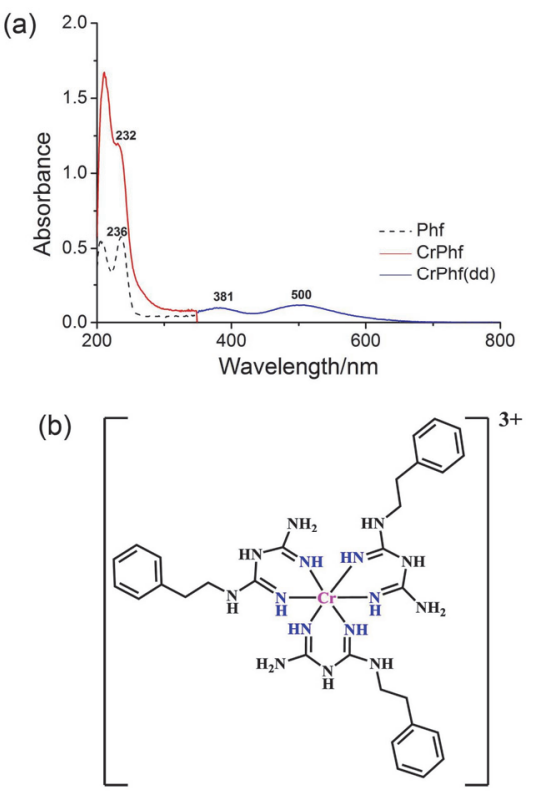

图 2 （a）苯乙双胍和配合物的吸收光谱, (b) 配合物结构

Figure 2 (a) Absorption spectra of $\mathrm{Phf}$ and $\mathrm{CrPhf}$, (b) structure of CrPhf. $[\mathrm{Phf}]=2.0 \times 10^{-5} \mathrm{~mol} \cdot \mathrm{L}^{-1},[\mathrm{CrPhf}]=4.0 \times 10^{-5} \mathrm{~mol} \cdot \mathrm{L}^{-1}$, $[\mathrm{CrPhf}(\mathrm{dd})]=2.0 \times 10^{-3} \mathrm{~mol} \cdot \mathrm{L}^{-1}$

\section{2 配合物的稳定性}

配合物的稳定性与其体内细胞的摄取、分布、作用 机制、代谢和生物活性及毒性等药理学性质紧密相关, 所以研究配合物的稳定性对探究其生物活性和机制非 常重要. $\mathrm{pH}$ 值和温度对溶液中配合物稳定性的影响是 研究生物活性比较重要的两个因素条件. 通过生理 $\mathrm{pH}$ 值和不同温度条件下的吸收光谱的变化推测配合物在 生物体内是否发生解离或水解, 这样可以为深入探究配 合物降糖降脂的作用机制和体内的转运情况，提供理论 指导. 实验是模拟三个生理环境, 现配制的配合物, 静 置在 $\mathrm{pH}$ 2.2(胃液)、7.4(体液)和 8.0 (小肠)溶液中, 或在 $25 \sim 60{ }^{\circ} \mathrm{C}$ 不同温度下 $(\mathrm{pH}=7.4$ 的 $\mathrm{PBS}$ 缓冲溶液)分别放 置 $15 \mathrm{~min}$ 后, 测定紫外-可见吸收光谱, 结果如图 3 所 示.

从图 3 上看, 配合物在 $\mathrm{pH}$ 从 2.2 到 8.0 的变化过程 中, 紫外区和 d-d 跃迁峰发生明显的红移, 可能是配合 物在从胃到小肠过程中慢慢发生了解离; 而配合物在不 同温度影响下, 紫外区的吸光度值随着温度的升高而增 大且轻微红移, 而 d-d 跃迁峰的变化不是很大, 说明配 合物在温度高于 $37{ }^{\circ} \mathrm{C}$ 开始发生解离. 因此, 在研究其 生物活性和其与蛋白、多肽结合作用时, 需要在体温条 件下进行实验.

\section{3 配合物的热分解性}

热分解性对于研究配合物的固态稳定性是又一重 要性质, 配合物的热分解在 Netzsch STA 2500 Regulus 综合热分析仪上进行, 热失重变化结果如图 4 所示.
图 4 热重和量热曲线可以看出, 配合物在 $100{ }^{\circ} \mathrm{C}$ 后 开始分解，并且热分解分 5 个过程完成，说明配合物的 固态稳定性也非常好.
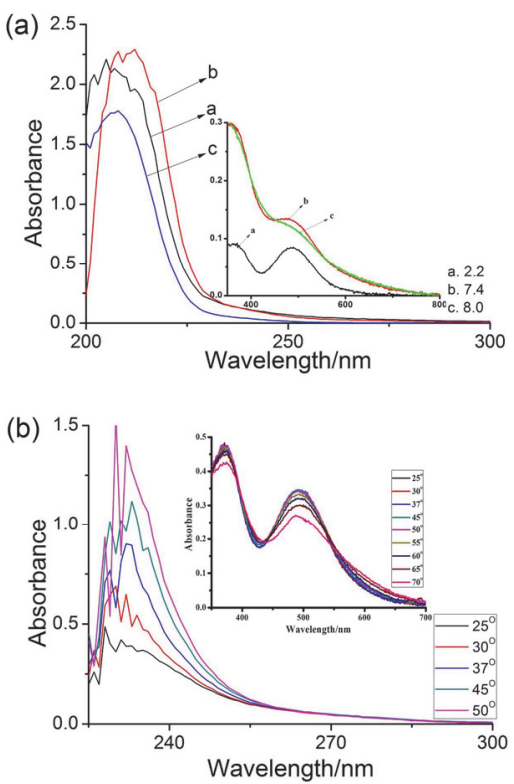

图 3 配合物在不同条件下的吸收光谱

Figure 3 Absorption spectra of complex under different conditions

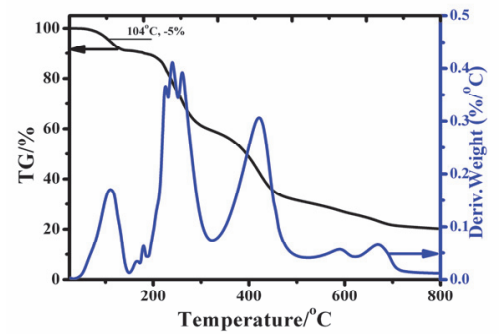

图 4 配合物的热重分析

Figure 4 Thermogravimetric analysis of complex

\section{4 配合物与双氧水的作用}

众所周知, $\mathrm{Cr}(\mathrm{VI})$ 有毒害可致癌 ${ }^{[19]}$, 因此, 在设计有 机铬(III)补充剂时, 对其氧化性的研究成为必需. 已有 诸多文献报道 $\mathrm{Cr}(\mathrm{III})$ 配合物在细胞外会被部分氧化为

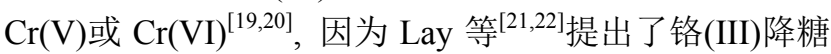
作用机制是在生理条件下 $\mathrm{Cr}(\mathrm{III})$ 被 $\mathrm{H}_{2} \mathrm{O}_{2}$ 或 $\mathrm{ClO}^{-}$氧化成 $\mathrm{Cr}(\mathrm{V})$ 或 $\mathrm{Cr}(\mathrm{VI})$, 然后在二硫苏糖醇 $(\mathrm{DTT})$ 还原剂作用下 抑制了蛋白质酪氨酸磷酸脂酶(PTPs)的活性或是阻止了 其表达, 从而推断 $\mathrm{Cr}(\mathrm{III})$ 的抗糖尿病性质可能与 $\mathrm{Cr}(\mathrm{V})$ 和 $\mathrm{Cr}(\mathrm{VI})$ 离子有间接关系. 本研究根据体内特征模拟了 三个生理条件: $\mathrm{pH}$ 为 7.4 缓冲溶液(PBS), 细胞培养液 (DMEM) 和人血清白蛋白(HSA), 分别配置浓度为 $1.0 \times$ $10^{-4} \mathrm{~mol} \cdot \mathrm{L}^{-1}$ 的配合物溶液, 各自移取 $1 \mathrm{~mL}$ 于 $\mathrm{EP}$ 管中, 加入 $2 \mu \mathrm{L} \mathrm{H}_{2} \mathrm{O}_{2}(30 \%)$, 静置在 $(37 \pm 0.5){ }^{\circ} \mathrm{C}$ 水浴中、反 应 $3.5 \mathrm{~h}$, 取出后摇匀, 加入 $1 \mathrm{~mL} 1,5$-二苯卡巴肼 $(0.01$ 
$\mathrm{g} \cdot \mathrm{mL}^{-1}$, 乙醇)指示剂, 再加入 $20 \mu \mathrm{L}$ 浓 $\mathrm{H}_{2} \mathrm{SO}_{4}$, 溶液立 即从无色变成了紫色(介质做空白), 在 $548 \mathrm{~nm}$ 下测其吸 光度 $A^{[23]}$, 结果如图 5 所示, 结果表明, 在未添加 $\mathrm{H}_{2} \mathrm{O}_{2}$ 时, 在各介质中, 仅少量 $\mathrm{Cr}(\mathrm{III})$ 被空气氧化为 $\mathrm{Cr}(\mathrm{VI})$, 比较稳定. 而添加了 $\mathrm{H}_{2} \mathrm{O}_{2}$ 后, $\mathrm{Cr}(\mathrm{III})$ 在各个生理条件下 都被氧化为 $\mathrm{Cr}(\mathrm{VI})$ 离子, 这与 Lay 等的实验结果相一致.
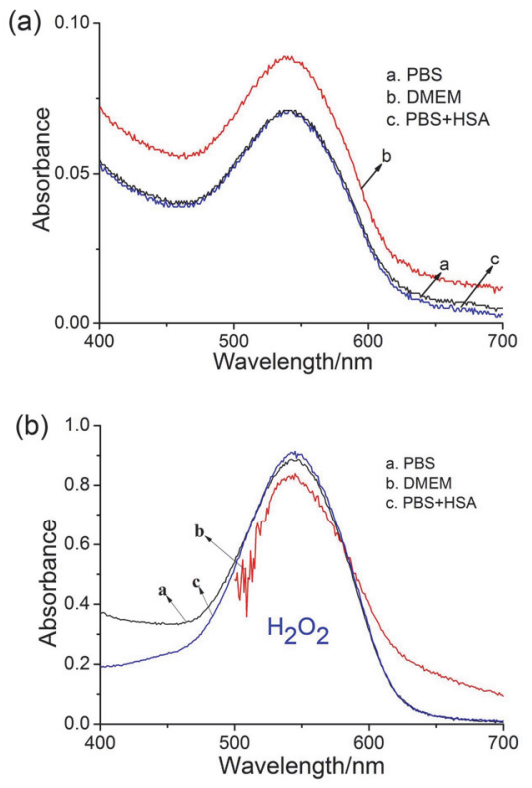

图 5 配合物在不同介质中加和不加 $\mathrm{H}_{2} \mathrm{O}_{2}$ 氧化后的吸收光谱

Figure 5 Absorption spectra of complex oxidized by $\mathrm{H}_{2} \mathrm{O}_{2}$ (with and wihout) in different media

\section{5 配合物的 SEM 和 XRD}

由于配合物经过多次结晶都得不到单晶, 因此, 测 试了配合物的扫描电镜 SEM 和 X 粉末衍射 XRD, 结果 如图 6 所示.

从扫描电镜图 6(a)上看, 配合物有比较好的均匀性, 得到了较清晰的大颗粒, 但形状不规则无形状. 粉末衍 射图 6(b,c)上, 配体峰形规则说明具有透明有序的晶型, 而形成的铬(III)配合物后, 就一个大包峰表明失去了配 体的晶形完全变为非晶态的化合物, 这也揭示出该配合 物不易获得可单晶测试的晶体.

\section{6 配合物的降糖活性}

本工作以二甲双胍为阳性对照物, 无机盐 $\mathrm{CrCl}_{3}$ 和 配合物对糖尿病模型鼠进行 12 周的干预实验, 结果见 表 1. 生化及代谢检测结果: 与对照组相比, 模型组及 干预组空腹血糖(FBG)、空腹胰岛素(FINS)、甘油三酯 (TG)、血清总胆固醇(TC)、高密度脂蛋白(HDL)和低密 度脂蛋白(LDL)显著增高, 差异有统计学意义 $(P<0.05)$; 与模型组相比, 干预组 FPG, FINS, TG 显著降低, 差异 有统计学意义 $(P<0.05)$; 与阳性对照物二甲双胍组比 较, 无机盐 $\mathrm{CrCl}_{3}$ 干预后的活性指标降低变化不是很显 著, 而配合物干预后的生物活性各项指标改善较明显,
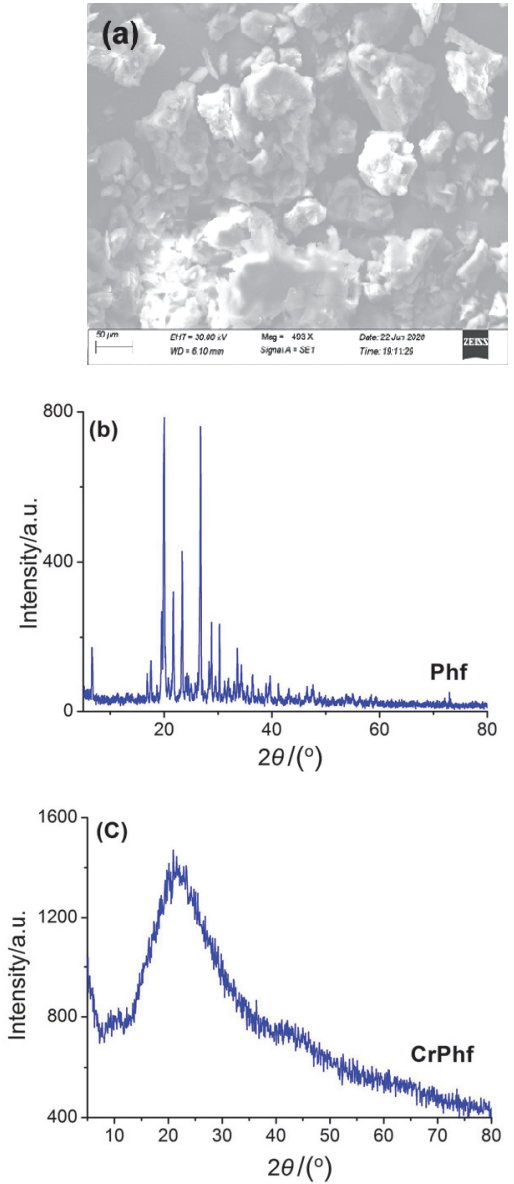

图 6 配合物的扫描电镜( $(a)$, 配体和配合物 $\mathrm{X}$ 粉末衍射图谱 $(\mathrm{b}, \mathrm{c})$ Figure 6 SEM photo of complex (a), XRD patterns of ligand and complex $(b, c)$

差异有统计学意义 $(P<0.05)$; HDL 和 LDL 的变化, 没有 显著的规律; 糖尿病与正常组小鼠的体重(BW)相比有 显著的增加，但是干预组并未对小鼠的 BW 造成明显的 改变，具体结果见表 1. 整体上，配合物对糖尿病小鼠 的生理指标的改善效果与预期的一样，这个可能与配合 物设计的结构和性质有较大的关系, 具体的药理作用机 制还有待进一步的实验论证. 同时，结果也与二甲双胍 铬的活性降糖结果一致 ${ }^{[24]}$.

\section{7 配合物的毒性评价}

\subsection{1 口服毒性}

药物分子口服毒性的评价通常是研究药物在体内 生物活性的第一步. 经过 12 周的化合物干预后, 没有出 现小鼠死亡. 实验结束后根据病理切片实验方法, 取出 小鼠胰腺、肝和肾部分组织经过染色处理后使用双目光 学显微镜观察其组织病理变化并拍照, 结果如图 7.

在图 7 中, 对照组与各实验组小鼠胰腺组织切面呈 现出正常的胰腺腺泡和导管细胞结构, 而且内分泌结构 和许多收集小管也是正常的. 关于肤腺的组织水肿和炎 症反应没有观察到. 配合物干预组的胰腺上可以看到一 
表 1 各组小鼠生化及代谢指标结果

Table 1 Results of biochemical and metabolic indexes of rats in each group

\begin{tabular}{|c|c|c|c|c|c|c|}
\hline & Week & Normal & Diabetic & $\mathrm{CrCl}_{3}$-treated & Met-treated & 1-treated \\
\hline \multirow{4}{*}{$\mathrm{BM} / \mathrm{g}$} & 0 & $19.24 \pm 1.16$ & $23.62 \pm 2.05$ & $24.14 \pm 2.14$ & $21.59 \pm 2.93$ & $22.92 \pm 1.86$ \\
\hline & 4 & $22.45 \pm 1.55$ & $30.5 \pm 2.40$ & $29.42 \pm 1.62$ & $28.64 \pm 0.82$ & $29.28 \pm 1.86$ \\
\hline & 8 & $29.35 \pm 1.48$ & $40.82 \pm 2.18$ & $37.89 \pm 3.01$ & $37.28 \pm 2.63$ & $39.17 \pm 1.77$ \\
\hline & 12 & $32.26 \pm 1.28$ & $48.26 \pm 3.00$ & $44.94 \pm 3.01$ & $43.08 \pm 3.11$ & $44.64 \pm 1.73$ \\
\hline \multirow{4}{*}{$\begin{array}{l}\mathrm{FBG} / \\
\left(\mathrm{mmol} \cdot \mathrm{L}^{-1}\right)\end{array}$} & 0 & $6.07 \pm 0.32^{*}$ & $11.59 \pm 0.39^{\#}$ & $11.18 \pm 0.61^{\#}$ & $13.47 \pm 0.57^{\#}$ & $12.45 \pm 0.62^{\#}$ \\
\hline & 4 & $5.54 \pm 0.23^{*}$ & $11.50 \pm 0.52^{\#}$ & $9.92 \pm 0.28^{\# *}$ & $9.95 \pm 0.52^{\#}$ & $9.83 \pm 0.40^{\#}$ \\
\hline & 8 & $5.60 \pm 0.20^{*}$ & $11.37 \pm 0.35^{\#}$ & $8.84 \pm 0.30^{\#^{*}}$ & $8.62 \pm 0.38^{\#}$ & $8.96 \pm 0.51^{\#}$ \\
\hline & 12 & $5.64 \pm 0.21^{*}$ & $11.70 \pm 0.58^{\#}$ & $8.76 \pm 0.35^{\# *}$ & $7.72 \pm 0.34^{\#}$ & $7.88 \pm 0.34^{\#}$ \\
\hline \multirow{4}{*}{$\begin{array}{l}\text { FINS/ } \\
\left(\mathrm{pmol} \mathrm{L}^{-1}\right)\end{array}$} & 0 & $12.36 \pm 1.50^{*}$ & $19.13 \pm 1.21^{\#}$ & $19.80 \pm 0.54^{\#}$ & $18.78 \pm 1.60^{\#}$ & $19.97 \pm 0.67^{\#}$ \\
\hline & 4 & $11.49 \pm 0.52^{*}$ & $21.71 \pm 0.52^{\#}$ & $17.23 \pm 0.91^{\# *}$ & $19.64 \pm 0.58^{\# *}$ & $18.22 \pm 0.65^{\#}$ \\
\hline & 8 & $11.25 \pm 0.34^{*}$ & $21.11 \pm 0.66^{\#}$ & $17.22 \pm 0.86^{\# *}$ & $18.40 \pm 0.58^{\# *}$ & $17.20 \pm 0.54^{\#}$ \\
\hline & 12 & $11.55 \pm 0.38^{*}$ & $19.89 \pm 0.46^{\#}$ & $15.99 \pm 0.76^{\# *}$ & $14.87 \pm 0.72^{\# *}$ & $15.28 \pm 0.59^{\#}$ \\
\hline \multirow{4}{*}{$\begin{array}{l}\mathrm{TC} / \\
\left(\mathrm{mmol} \cdot \mathrm{L}^{-1}\right)\end{array}$} & 0 & $1.92 \pm 0.15^{*}$ & $4.66 \pm 0.64^{\#}$ & $4.28 \pm 0.32^{\#}$ & $4.41 \pm 0.74^{\#}$ & $4.45 \pm 0.34^{\#}$ \\
\hline & 4 & $1.74 \pm 0.12^{*}$ & $4.17 \pm 0.32^{\#}$ & $3.77 \pm 0.19^{\#}$ & $3.90 \pm 0.26^{\#}$ & $3.34 \pm 0.20^{\#}$ \\
\hline & 8 & $1.92 \pm 0.093^{*}$ & $4.68 \pm 0.23$ & $3.50 \pm 0.24^{\# *}$ & $3.68 \pm 0.20^{\#}$ & $3.08 \pm 0.19^{\#}$ \\
\hline & 12 & $1.95 \pm 0.083^{*}$ & $4.14 \pm 0.24^{\#}$ & $3.66 \pm 0.28^{\#}$ & $3.51 \pm 0.20^{\#}$ & $2.88 \pm 0.22^{\#}$ \\
\hline \multirow{4}{*}{$\begin{array}{l}\mathrm{TG} / \\
\left(\mathrm{mmol} \cdot \mathrm{L}^{-1}\right)\end{array}$} & 0 & $0.55 \pm 0.050$ & $1.63 \pm 0.22$ & $2.02 \pm 0.19$ & $1.77 \pm 0.22$ & $1.79 \pm 0.12$ \\
\hline & 4 & $0.50 \pm 0.043^{*}$ & $1.92 \pm 0.21^{\#}$ & $1.87 \pm 0.20^{*}$ & $1.70 \pm 0.22^{\#}$ & $1.49 \pm 0.11^{\#}$ \\
\hline & 8 & $0.52 \pm 0.026^{*}$ & $2.04 \pm 0.21^{\#}$ & $1.63 \pm 0.14^{*}$ & $1.72 \pm 0.11^{\#}$ & $1.29 \pm 0.12^{\#}$ \\
\hline & 12 & $0.53 \pm 0.029^{*}$ & $2.41 \pm 0.23^{\#}$ & $1.69 \pm 0.24^{*}$ & $1.67 \pm 0.15^{*}$ & $1.21 \pm 0.13^{\#}$ \\
\hline \multirow{4}{*}{$\begin{array}{l}\text { HDL/ } \\
\left(\mathrm{mmol} \cdot \mathrm{L}^{-1}\right)\end{array}$} & 0 & $1.31 \pm 0.09^{*}$ & $0.90 \pm 0.099^{\#}$ & $0.87 \pm 0.062^{\#}$ & $0.82 \pm 0.070^{\#}$ & $0.91 \pm 0.07^{\#}$ \\
\hline & 4 & $1.24 \pm 0.092^{*}$ & $0.80 \pm 0.064^{\#}$ & $0.98 \pm 0.059^{\#}$ & $0.86 \pm 0.081^{\#}$ & $0.96 \pm 0.053^{\#}$ \\
\hline & 8 & $1.19 \pm 0.086^{*}$ & $0.75 \pm 0.040^{\#}$ & $0.86 \pm 0.049^{\#}$ & $0.86 \pm 0.080^{\#}$ & $0.89 \pm 0.056^{\#}$ \\
\hline & 12 & $1.21 \pm 0.081^{*}$ & $0.79 \pm 0.033^{\#}$ & $0.90 \pm 0.050^{\#}$ & $0.84 \pm 0.080^{\#}$ & $0.94 \pm 0.052^{\#}$ \\
\hline \multirow{4}{*}{$\begin{array}{l}\mathrm{LDL} / \\
\left(\mathrm{mmol} \cdot \mathrm{L}^{-1}\right)\end{array}$} & 0 & $0.75 \pm 0.064$ & $0.87 \pm 0.050$ & $0.85 \pm 0.095$ & $0.80 \pm 0.061$ & $1.02 \pm 0.06$ \\
\hline & 4 & $0.68 \pm 0.027^{*}$ & $0.99 \pm 0.064^{\#}$ & $0.76 \pm 0.052^{*}$ & $0.81 \pm 0.050^{\#}$ & $0.83 \pm 0.053^{\#}$ \\
\hline & 8 & $0.70 \pm 0.024^{*}$ & $1.09 \pm 0.062^{\#}$ & $0.81 \pm 0.066^{*}$ & $0.81 \pm 0.050^{\#}$ & $0.81 \pm 0.05^{\#}$ \\
\hline & 12 & $0.71 \pm 0.028^{*}$ & $0.99 \pm 0.054^{\#}$ & $0.84 \pm 0.040^{\#}$ & $0.8 \pm 0.036^{\#}$ & $0.80 \pm 0.034^{\#}$ \\
\hline
\end{tabular}

些粘液, 是胰腺导管分泌的蛋白液, 属于正常分泌物. 小鼠肾脏的组织切片也全部呈现为正常结构的间质组 织，既无炎症反应，也没有肾间质纤维化的反应. 最后， 小鼠的肝脏中央静脉、肝细胞索、汇管区等结构清晰, 同 时也没有观察到轻度单核细胞的过滤和肝细胞结构的 轻度损伤. 从病理切片实验结果看, 铬和其配合物对胰 腺、肝脏和肾脏均没有影响, 初步估计苯乙双胍铬配合 物对生物机体是无伤害的.

\subsubsection{MTT 分析}

使用酶标分析仪测试了细胞的活性, 其结果如图 8 . $1 \%$ DMSO 被作为对照, $\mathrm{CrCl}_{3}$ 、 $\mathrm{Phf}$ 和三个浓度的 $\mathrm{CrPhf}$. 结果证明，除了高浓度配合物作用后的存活率低于 $80 \%$ 以下, 其它两个浓度的细胞的存活率都高于 $95 \%$, 表明 配合物 CrPhf 对 MCF-7 cells 是无毒的且生物相容性也 是很好的.

\section{8 配合物与胰高血糖素作用}

为了探索配合物的生物降糖机制, 我们选取胰高血 糖素(glucagon)为对象, 通过苂光光谱法研究与配合物 的结合作用. Glucagon 是一种由胰脏胰岛 $\alpha$-细胞分泌的 激素, 与胰岛素的生物学功能恰好相反, 因此又称抗胰 岛素. Glucagon 经过 cAMP-PK 系统, 激活肝细胞的磷酸 化酶, 加速糖原分解. 若 glucagon 分泌过剩了, 就会表 现出高血糖症，因此有效抑制 glucagon 的过量分泌，可

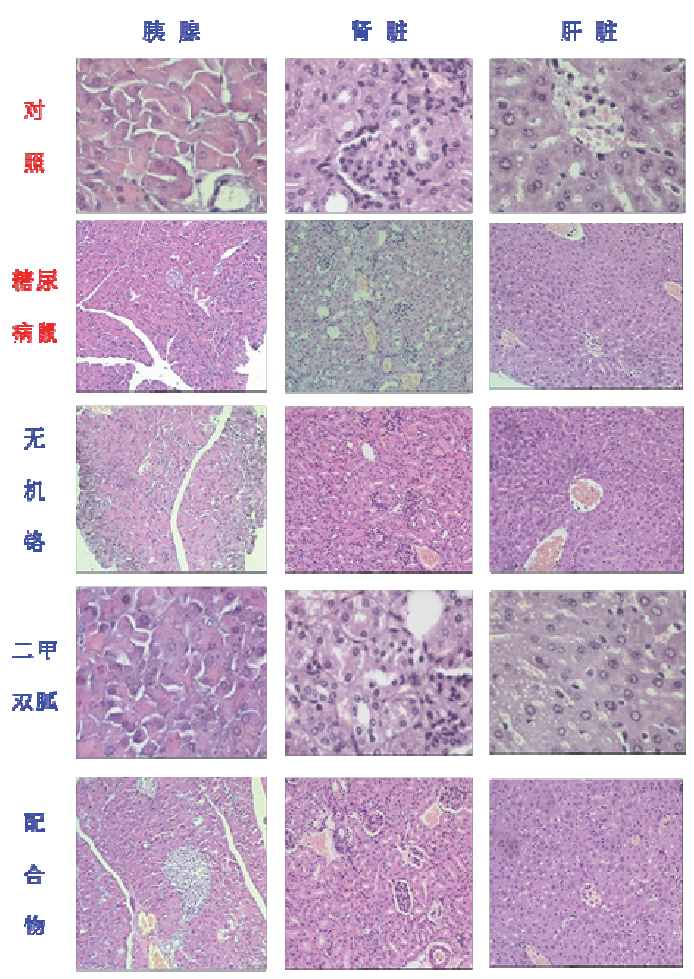

图 $7 \mathrm{CrCl}_{3} 、$ Met 和配合物干预后的胰腺、肝和肾脏部分病理组织图 像

Figure 7 Histopathological images of pancreas, kidney and liver sections treated $\mathrm{CrCl}_{3}$, Met and complex 


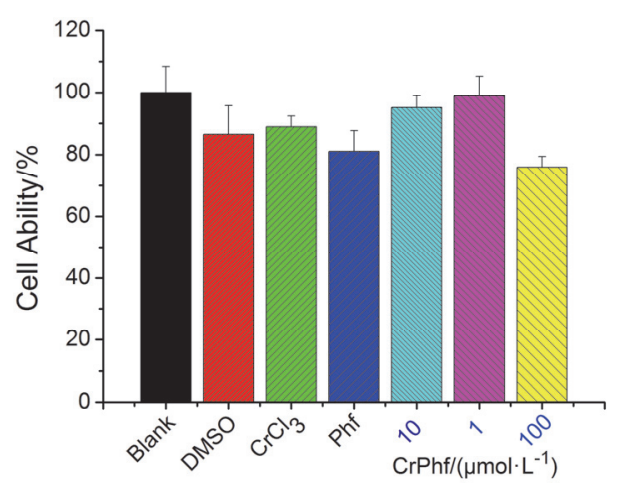

图 8 配合物对细胞的毒性作用. 通过 MTT 试验, 苯乙双胍 Phf (10 $\left.\mu \mathrm{mol} \cdot \mathrm{L}^{-1}\right)$ 和配合物 $\mathrm{CrPhf}\left(1,10,100 \mu \mathrm{mol} \cdot \mathrm{L}^{-1}\right)$ 对 MCF-7 癌细胞 $(2.5 \times$ $10^{4}$ cells $\left./ \mathrm{mL}\right)$ 作用的细胞毒性评价, 体积比为 $1 \%$ DMSO 作为对照 $(P$ $<0.05)$, 结果是通过 6 次平行实验的土标准偏差.

Figure 8 Cytotoxic effect of complex on cell lines. The cytotoxicity of $\operatorname{Phf}\left(10 \mu \mathrm{mol} \cdot \mathrm{L}^{-1}\right)$ and complex $\left(1,10,100 \mu \mathrm{mol} \cdot \mathrm{L}^{-1}\right)$ were evaluated on MCF-7 cell lines $\left(2.5 \times 10^{4}\right.$ cells $\left./ \mathrm{mL}\right)$ by the MTT assay for $24 \mathrm{~h}$. $1 \%$ DMSO $(V / V)$ was used as a control experiment $(P<0.05)$. Results are mean \pm SD of six independent experiments.

能有助于防止糖尿病的发病. 临床已公认, 糖尿病是由 于 $\alpha$ 细胞分泌 glucagon 的过量和胰岛 $\beta$ 细胞分泌胰岛素 的不足所共同导致, glucagon 成为治疗 II 型糖尿病的新 靶点 ${ }^{[15]}$. Glucagon 的分子结构中含有 1 个色氨酸和 2 个 酪氨酸残基使其具有内源性苂光, 而色氨酸残基的内源 荧光通常被用作蛋白分子与化合物作用的有效光谱探 针.

在温度 $(37 \pm 0.5){ }^{\circ} \mathrm{C}$, 设定激发和发射光栅狭缝宽 均为 $5 \mathrm{~nm}$, 用 $280 \mathrm{~nm}$ 激发 glucagon 的荧光光谱, glucagon(浓度为 $1.0 \times 10^{-5} \mathrm{~mol} \cdot \mathrm{L}^{-1}$ ) 苂光发射峰位置在 347 $\mathrm{nm}$ 左右附近, 每次 $10 \mu \mathrm{L}$ 化合物 $\left(1.0 \times 10^{-4} \mathrm{~mol} \cdot \mathrm{L}^{-1}\right)$ 对 glucagon 滴定, 结果如图 9 和图 S5 所示. 从图上看, glucagon 在 $347 \mathrm{~nm}$ 的荧光发射峰强度随着化合物的逐 渐滴加呈现出规律性地猝灭, 且最大峰位置无明显移 动. 从内插图可以看出, $\left[\mathrm{CrCl}_{3}\right]$ 和 $[\mathrm{CrPhf}]$ 对 glucagon 的 荧光猝灭平衡时的化学计量比约为 $2: 1$, 说明 $\mathrm{Cr}$ (III)对 glucagon 有一定的结合作用, 形成了不发荧光的 glucagon-配合物复合物后, glucagon 荧光发生猝灭.

各种大分子蛋白质、多肽等荧光体与荧光猝灭剂分 子间的猝灭机理可由 Stern-Volmer 方程(1)和(2)进行阐 述; 若化合物与 glucagon 结合形成不发荧光的基态复合 物, 根据公式(3)可以得到化合物与 glucagon 复合物的 结合常数, 公式推导见文献[25,26], 则有:

$$
\begin{aligned}
& F_{0} / F=1+K_{\mathrm{q}} \tau_{0}[\mathrm{Q}]=1+K_{\mathrm{sv}}[\mathrm{Q}] \\
& K_{\mathrm{sv}}=K_{\mathrm{q}} \tau_{0} \\
& {[\mathrm{~S}]_{\mathrm{t}} /[\mathrm{P}]_{\mathrm{b}}=F_{0} /\left([\mathrm{P}]_{\mathrm{t}} F\right) K+n}
\end{aligned}
$$

式中, $F_{0}$ 和 $F$ 分别为不加和加入化合物猝灭剂时, glucagon 的苂光强度; [Q] 代表猝灭剂浓度; $K_{\mathrm{sv}}$ 为 Stern-Volmer 动态猝灭常数, 通过 $F_{0} / F$ 对 $[\mathrm{Q}]$ 作图拟合得 到; $K_{\mathrm{q}}$ 为生物大分子的猝灭速率常数; $\tau_{0}$ 为生物大分子 (a)

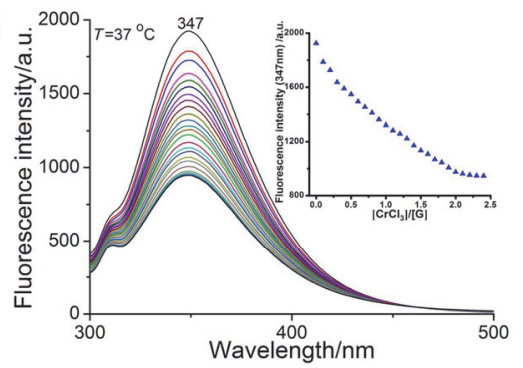

(b)

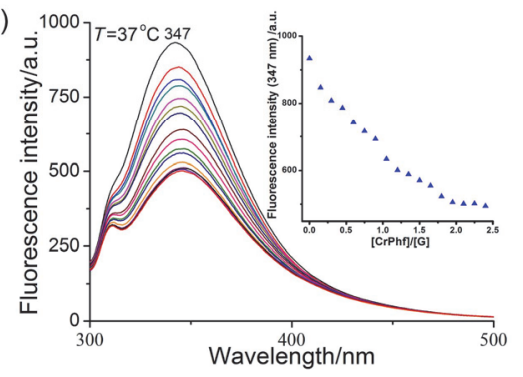

图 9 化合物对胰高血糖素苂光光谱(内插图: 在 $347 \mathrm{~nm}$ 处苂光强度 变化)

Figure 9 Fluorescence spectra of glucagon at different concentrations of complex (Insert: the changes of fluorescence at $347 \mathrm{~nm}$ )

在没有添加猝灭剂时的荧光平均寿命; 利用图 9 中化合 物对 glucagon 苂光猝灭的变化数值, 分别作出 glucagon 的 Stern-Volmer 曲线(图 10(a, b) 和图 S6(a)), 并对实验数 据线性拟合得到 $K_{\mathrm{SV}}$, 接着根据公式(2)计算得到 $K_{\mathrm{q}}$, 结 果见表 $2 ;[\mathrm{S}]_{\mathrm{t}}$ 为化合物的总浓度, $[\mathrm{P}]_{\mathrm{t}}$ 表示 glucagon 的总 浓度, $[\mathrm{P}]_{\mathrm{b}}$ 为结合有化合物的 glucagon 浓度, $n$ 为结合位 点数, $K$ 为化合物与 glucagon 的结合常数, 以 $[\mathrm{S}]_{\mathrm{t}} /[\mathrm{P}]_{\mathrm{b}}$ 对 $F_{0} /\left([\mathrm{P}]_{\mathrm{t}} F\right)$ 作图, 见图 10(c, d) 和图 S6(b), 由图中直线的 斜率倒数可得结合常数 $K$, 结果见表 2 .
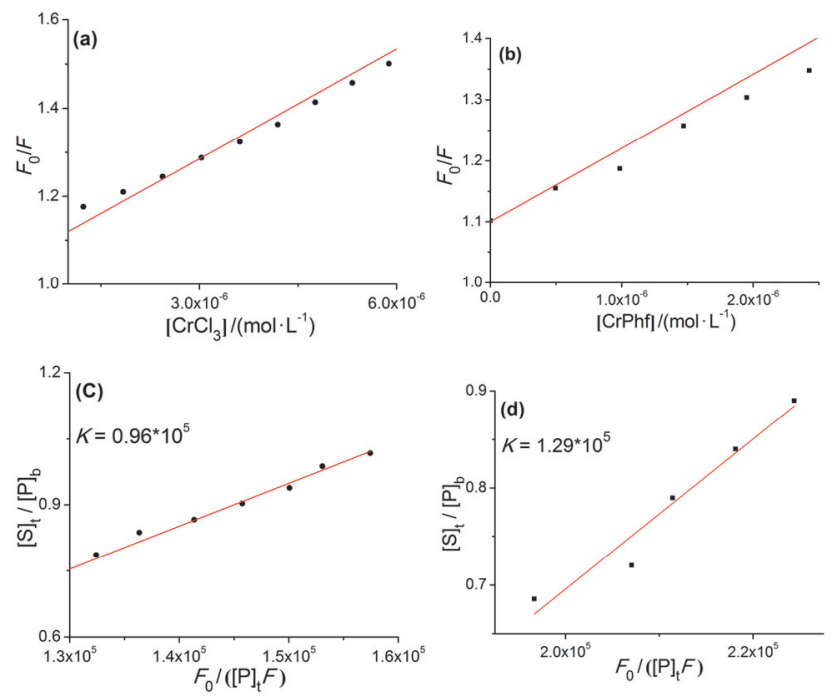

图 10 在 $37{ }^{\circ} \mathrm{C}$ 下化合物滴定胰高血糖素的 Stern-Volmer 曲线 $(\mathrm{a}, \mathrm{b})$ 和 Scatchard 图(c, d)

Figure 10 Stern-Volmer (a, b) and Scatchard (c, d) plots of fluorescence quenching of glucagon by compound at $37{ }^{\circ} \mathrm{C}$ 
已知苂光猝灭剂对生物大分子的最大猝灭值 $K_{\mathrm{q}}$ 为 $2.0 \times 10^{10} \mathrm{~L} \cdot \mathrm{mol}^{-1} \cdot \mathrm{s}^{-1}$ ，从表 2 的数据看, $\mathrm{CrCl}_{3}, \mathrm{Cr}\left(\mathrm{NH}_{3}\right)_{6}$ 和 CrPhf两个化合物对 glucagon 的 $K_{\mathrm{q}}$ 都远大于 $2.0 \times 10^{10}$ $\mathrm{L} \cdot \mathrm{mol}^{-1} \cdot \mathrm{s}^{-1}$, 由此推断, 化合物对 glucagon 的作用主要 是化合物中的 $\mathrm{Cr}$ (III)与 glucagon 结合而发生静态猝灭.

表 2 化合物与胰高血糖素在 $37{ }^{\circ} \mathrm{C}$ 时的猝灭常数和结合常数

Table 2 Quenching and binding constant $K$ of compound with glucagon at $37{ }^{\circ} \mathrm{C}$

\begin{tabular}{cccc}
\hline 配合物 & $K_{\mathrm{sv}} /\left({\left.\mathrm{L} \cdot \mathrm{mol}^{-1}\right)}^{-1}\right.$ & $K_{\mathrm{q}} /\left({\left.\mathrm{L} \cdot \mathrm{mol}^{-1} \cdot \mathrm{s}^{-1}\right)} K /\left(\mathrm{L} \cdot \mathrm{mol}^{-1}\right)\right.$ \\
\hline$\left[\mathrm{CrCl}_{3}\right]$ & $7.09 \times 10^{5}$ & $7.09 \times 10^{13}$ & $0.96 \times 10^{5}$ \\
{$[\mathrm{CrPhf}]$} & $10.28 \times 10^{5}$ & $10.28 \times 10^{13}$ & $1.29 \times 10^{5}$ \\
{$\left[\mathrm{Cr}\left(\mathrm{NH}_{3}\right)_{6}\right]$} & $8.83 \times 10^{5}$ & $8.83 \times 10^{13}$ & $1.32 \times 10^{5}$ \\
\hline
\end{tabular}

表 2 中配合物 $\mathrm{CrPhf}$ 和 $\mathrm{Cr}\left(\mathrm{NH}_{3}\right)_{6}$ 与 glucagon 的结合 常数大于 $10^{5}$, 而无机盐 $\mathrm{CrCl}_{3}$ 与 glucagon 小于 $1.0 \times 10^{5}$ $\mathrm{L} \cdot \mathrm{mol}^{-1}$, 表明配合物与 glucagon 有较强结合能力, 可能 是由于配合物在转化吸收的过程中形成了中间体 $\mathrm{Cr}\left(\mathrm{NH}_{3}\right)_{6}$, 更易与 glucagon 相结合作用.

\section{9 配合物降糖机理}

经过以上的综合分析, 拟推断苯乙双胍铬的生物降 糖机制还有可能如图式 1 所示的作用机理. 苯乙双胍铬 从进入胃部到小肠的过程中, 逐渐发生解离, 中间形成 了六氨合铬(III)离子 $\mathrm{Cr}\left(\mathrm{NH}_{3}\right)_{6}$, 然后可以与过剩的胰高 血糖素进行结合作用形成了胰高血糖素与铬的复合物, 从而有效降低血糖含量达到缓解 II 型糖尿病症的目的. 同时, 苯乙双胍本身就具有降糖的活性, 那么二者的结 合应该可以达到协同生物学效应.

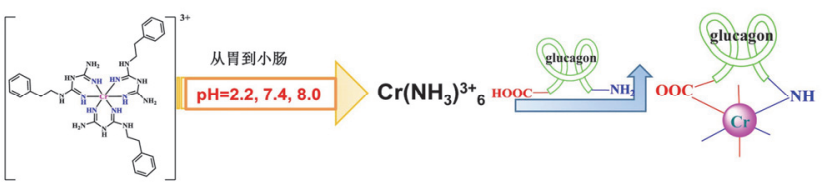

图式 1 苯乙双胍铬在体内可能的作用机理

Scheme 1 The proposed mechanism of $\mathrm{CrPhf}$ in vivo

\section{3 结论}

以具有抗糖尿病功效的药物苯乙双胍为配体, 制备 了苯乙双胍铬(III)配合物, 对其结构进行了表征, 并研 究了配合物的稳定性、与 $\mathrm{H}_{2} \mathrm{O}_{2}$ 的反应性和形貌. 构建了 糖尿病 C57 小鼠模型, 研究了其生物活性和毒性. 在此 基础上, 通过光谱法研究了配合物与胰高血糖素的结合 作用, 得到结合常数值为 $1.29 \times 10^{5} \mathrm{~L} \cdot \mathrm{mol}^{-1}$, 结合位点 约为 1 . 推断配合物与胰高血糖素按 $1: 1$ 结合形成复合 物，从而抑制了过剩 glucagon 的表达，达到降糖的目的. 说明配合物在 II 型糖尿病的预防和治疗产生了有益的 效果, 对机体组织也是无毒的. 配合物的设计不但保留 了苯乙双胍的降糖活性, 而且也丰富了金属配合物的多 功能化应用, 进而为新型双胍类药物的研发开辟了新思 路. 同时, 配合物 $\mathrm{CrPhf}$ 的研发也有可能为糖尿病的辅
助治疗提供一个新方向.

\section{4 实验部分}

\section{1 苯乙双胍铬(III)配合物的合成}

称取 $18.614 \mathrm{~g}(\approx 90 \mathrm{mmol})$ 苯乙双胍 $(\mathrm{Phf})$ 于圆底烧 瓶, 加入 $0.410 \mathrm{~g}(\approx 100 \mathrm{mmol}) \mathrm{NaOH}$, 再加入 $150 \mathrm{~mL}$ $\mathrm{CH}_{3} \mathrm{OH}$, 室温下快速搅拌、滤掉固体 $\mathrm{NaCl}$; 然后在慢速 搅拌下将 $8.014 \mathrm{~g}(\approx 30 \mathrm{mmol}) \mathrm{CrCl}_{3} \cdot 6 \mathrm{H}_{2} \mathrm{O}$ 的水溶液慢 慢滴入到 $\mathrm{Phf}$ 的滤液中, 在室温下继续搅拌 $1 \mathrm{~h}$, 逐渐得 到大量红色固体, 停止搅拌, 静置 $2 \mathrm{~h}$, 过滤, 用少量乙 醇、乙醚洗涤后，自然晾干得到红色粉末固体，产率约 $49.2 \%$ (以 $\mathrm{Cr}$ 量计算).

\subsection{T2DM 小鼠动物模型的建立、实验分组及生化指 标的测试}

在山西医科大学动物中心购置 20 30 g 健康 C57 小鼠 60 多只, 其中 50 多只用高脂高糖饲料喂养四周, 然后向每只小鼠的尾静脉注射链脲霉素(STZ, $50 \mathrm{mg} ・$ $\mathrm{kg}^{-1}$ 体重), 继续用高糖高脂饲料再喂养四周, 通过尾部 采血检测每只小鼠的空腹血糖(FBG), 所有小鼠 FBG 水 平大于 $11.1 \mathrm{mmol} \cdot \mathrm{L}^{-1}$, 建模成功.

将 50 只糖尿病鼠随机分为 4 组, 与 12 只正常小鼠 单笼饲养，标准屏蔽环境，相对湿度 $55 \%$ ，昼夜交替. 在实验期间所有的小鼠可以自由喝水及进食, 干预组小 鼠每日早上以口服方式按 $1.0 \mathrm{mg} \mathrm{Cr} / \mathrm{kg}$ (小鼠体重)剂量 灌胃给药, 对照组及模型组每日空腹灌胃生理盐水 1.5 $\mathrm{mL}$. 小鼠实验为期 12 周, 每四周全自动生化仪检测每 只小鼠的空腹血糖(FBG)、空腹胰岛素(FINS)、甘油三 酯(TG)、血清总胆固醇(TC)、高密度脂蛋白(HDL)和低 密度脂蛋白(LDL), 同时记录小鼠的体重(BW)变化. 实 验经伦理委员会批准, 实验过程中严格人道对待动物.

\section{3 实验动物组织病理学检查}

经过药物干预实验后, 每组实验动物各取两只 $12 \mathrm{~h}$ 禁食禁饮后被 $\mathrm{CO}_{2}$ 窒息法处死，接着小鼠的肝、肾和胰 腺被迅速摘除，用生理盐水清洗，全自动脱水机脱水后 烘干，切 $5 \mu \mathrm{m}$ 片并 HE 染色后蜡封，待用. 按照临床实 验方法, 在显微镜下观察切片组织器官结构、损伤、病 变和感染的情况.

\section{References}

[1] Yu, J. C.; Wang, J. Q.; Zhang, Y. Q.; Chen, G. J.; Mao, W.; Ye, Y. Q.; Kahkoska, A. R.; Buse, J. B.; Langer, R.; Gu, Z. Nat. Biomed. Eng. 2020, 4, 499 .

[2] Williamson, R. M.; Price, J. F.; Glancy, S.; Perry, E.; Nee, L. D.; Hayes, P. C.; Frier, B. M.; Van Look, L. A. F.; Johnston, G. I.; Reynolds, R. M.; Mark, W. J. Diabetes Care. 2011, 34, 1139.

[3] Samuel, V. T.; Liu, Z. X.; Qu, X. Q.; Elder, B. D.; Bilz, S.; Befroy, D.; Romanelli, A. J.; Shulman, G. I. J. Biol. Chem. 2004, 279 , 32345 .

[4] Xu, B.; Broome, U.; Uzunel M.; Nava, S.; Ge, X. P.; Kumagai-Braesch, M.; Hultenby, K.; Christensson, B.; Ericzon, B.; Holgersson, J.; Sumitran-Holgersson, S. Am J. Pathol. 2003, 163, 
1275 .

[5] Farrell, G. C.; Chitmri, S.; Lau, G. K.; Sollano, J. D. J. Gastroen Hepatol. 2007, 22, 775.

[6] Hidenobu, S.; Tomoyo, N.; Yusuke, I.; Satoru, H.; Ryoma, N.; Makoto, O. Molecules 2019, 24, 3131.

[7] Evans, J. L.; Maddux, B. A.; Goldfine, I. D. Antioxid. Redox. Sign. 2005, 7, 1040

[8] Pechova, A.; Pavlata, L. Vet Med. 2007, 52, 1.

[9] Hua, Y. A.; Clark, S.; Ren, J.; Sreejayan, N. J. Nutr. Biochem. 2012, 23, 313 .

[10] Yang, X. P.; Palanichamy, K.; Ontko, A. C.; Rao, M. N. A.; Fang, C. X.; Ren, J.; Sreejayan, N. FEBS Lett. 2005, 579, 1458.

[11] Duan, W. S.; Liu, B.; Sun, Z. G.; Yang, B. S. Acta Chim. Sinica 2011，69，1789 (in Chinese). (段文胜, 刘斌, 孙占国, 杨斌盛, 化 学学报, 2011, 69, 1789.)

[12] Nan, J. X.; Liu, B.; Yang, B. S. Acta Chim. Sinica 2011, 69, 640 (in Chinese). (南俊霞, 刘斌, 杨斌盛, 化学学报, 2011, 69, 640.)

[13] Liu, B.; Sun, Z. G.; Yang, B. S. Acta Chim. Sinica 2008, 66, 2353 (in Chinese). (刘斌, 孙占国, 杨斌盛, 化学学报, 2008, 66, 2353.)

[14] Liu, B.; Li, Y. Q.; Yang, B. S. Acta Chim. Sinica 2006, 64, 917 (in Chinese). (刘斌, 李英奇, 杨斌盛, 化学学报, 2006, 64, 917.)
[15] Cho, Y. M.; Merchant, C. E.; Kieffer, T. J. Pharmacol. Therapeut. 2012, 135, 247.

[16] Nakamoto, K. Infrared and Raman Spectra of Inorganic and Coordination Compounds, Part B, 5th ed., Wiley, New York, 1997.

[17] Adam, A. M. A.; Sharshar, T.; Mohamed, M. A.; Ibrahim, O. B.; Refat, M. S. Spectrochim. Acta A 2015, 149, 323.

[18] Hamada, Y. Z.; Carlson, B. L.; Shank, J. T. Synth. React. Inorg. Met. Org. Chem. 2003, 33, 1425.

[19] Chen, J. M.; Hao, O. J. Crit. Rev. Env. Sci. Tec. 1998, 28, 219.

[20] Andersen, J. E. T. Anal. Chim. Acta 1998, 361, 125.

[21] Irma, M.; Aviva, L.; Lay, P. A. Angew. Chem. Int. Ed. 2004, 43, 4504

[22] Levina, A.; Lay, P. A. Coord. Chem. Rev. 2005, 249, 281.

[23] Pettine, M.; Lanoce, T.; Liberatori, A.; Loreti, L. Anal. Chim. Acta 1988, 209, 315

[24] Dong, J. L.; Liu, B.; Liang, G.; Yang, B. S. J. Coord. Chem. 2018, 4, 1526.

[25] Yang, B. S.; Yang, P. Chin. Sci. Bull. 1984, 29, 1066 (in Chinese). (杨斌盛, 杨频, 科学通报, 1984, 29, 1066.)

[26] Shrivastava, H. Y.; Nair, B. U. J. Inorg. Biochem. 2004, 98, 991.

(Cheng, B.) 